\title{
Accretion rate and the occurrence of multi-peaked X-ray bursts
}

\author{
A. L. Watts and I. Maurer
}

\begin{abstract}
Max Planck Institut für Astrophysik, Karl-Schwarzschild-Str. 1, 85741 Garching, Germany
e-mail: anna@mpa-garching.mpg.de
\end{abstract}

Received 18 February 2007 / Accepted 26 March 2007

\section{ABSTRACT}

\begin{abstract}
Most Type I X-ray bursts from accreting neutron stars have a lightcurve with a single peak, but there is a rare population of faint bursts that are double or even triple peaked. Suggested mechanisms include polar ignition with equatorial stalling, or multi-step energy release; the latter being caused by hydrodynamic instabilities or waiting points in the nuclear reaction sequence. We present an analysis of the accretion rate dependence of the multi-peak bursts, and discuss the consequences for the various models. The observations pose particular challenges for the polar ignition mechanism given current models of ignition latitude dependence.
\end{abstract}

Key words. binaries: general - stars: individual: 4U 1636-536 - stars: neutron - stars: rotation - X-rays: bursts - X-rays: stars

\section{Introduction}

Neutron stars in Low Mass X-ray binaries (LMXBs) accrete hydrogen $(\mathrm{H})$ or helium $(\mathrm{He})$ rich material from their companion stars via Roche lobe overflow. This accumulating fuel burns and under certain circumstances the burning process becomes unstable, resulting in a thermonuclear flash known as a Type I X-ray burst (for recent reviews see Strohmayer \& Bildsten (2006); Galloway et al. (2007)). The nature of the burning depends primarily on accretion rate (Fujimoto et al. 1981; Fushiki \& Lamb 1987; Bildsten 1998; Narayan \& Heyl 2003; Cooper $\&$ Narayan 2006). At the lowest accretion rates, bursts are triggered by unstable $\mathrm{H}$ burning. Above $\sim 1 \%$ of the Eddington accretion rate $\left(\dot{M}_{\text {Edd }} \sim 1 \times 10^{-8} M_{\odot} \mathrm{yr}^{-1}\right)$, H burning stabilises. A shell of He builds up and this triggers pure He bursts. Above $\sim 10 \% \dot{M}_{\text {Edd }}$, not all of the $\mathrm{H}$ can burn before the He triggers, leading to various classes of mixed $\mathrm{H} / \mathrm{He}$ bursts. Eventually, above $\sim 30 \% \dot{M}_{\text {Edd }}$, He burning also stabilises and no more bursts are seen. Observations support the existence of this upper limit (Cornelisse et al. 2003; Galloway et al. 2007).

The vast majority of X-ray bursts have a single peak in the lightcurve. However, there are some with a multi-peaked structure. These fall into two classes. The first are Photospheric Radius Expansion (PRE) bursts, where the flux reaches the Eddington luminosity. As the envelope is lifted from the star the temperature drops and the energy of the emitted photons falls below the X-ray band, leading to an apparent drop in countrate. As the envelope contracts again the temperature rises, and there is a second peak in X-ray emission (Paczyński 1983). If one looks at the bolometric rather than the X-ray luminosity, the double-peak structure vanishes.

In this Letter we focus on the second class of multi-peaked bursts. These bursts have lower peak countrates and do not involve PRE, so that the multi-peak structure is also visible in the bolometric luminosity. Double-peaked bursts have been reported for 4U 1608-52 (Penninx et al. 1989), GX 17+2 (Kuulkers et al. 2002) and 4U 1709-267 (Jonker et al. 2004). The largest sample, however, comes from 4U 1636-536. Observations with EXOSAT revealed 5 double-peaked bursts and one triple-peaked
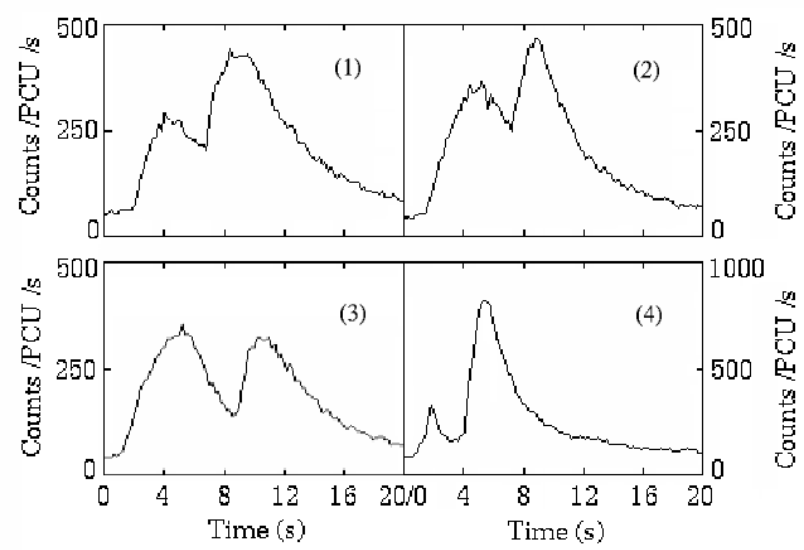

Fig. 1. 2-25 keV X-ray lightcurves for the four non-PRE multi-peaked bursts in the RXTE archive for 4U 1636-536. Burst numbers (1)-(4) are burst numbers 40, 48, 56 and 111 respectively in the burst catalogue of Galloway et al. (2007). PCU refers to the RXTE Proportional Counter Units. The burst modelled in Bhattacharyya \& Strohmayer (2006a) is burst (3); that in Bhattacharyya \& Strohmayer (2006b) is burst (4).

burst (Sztajno et al. 1985; van Paradijs et al. 1986; Lewin et al. 1987). Subsequent observations with the Rossi X-ray Timing Explorer (RXTE) have added four more double-peaked bursts (Fig. 1, see also Galloway et al. (2007)).

Since the discovery of these bursts, a number of causes have been suggested. Fisker et al. (2004) give an excellent review of the suggested processes and the observational results that have led to some being ruled out. The most likely mechanisms that remain viable involve a stepped release of thermonuclear energy. This could be caused by hydrodynamical instabilities leading to mixing (Fujimoto et al. 1988) or, as suggested by Fisker et al. (2004), a waiting point that impedes the nuclear reaction sequence.

More recent work has suggested that the critical factor may be the latitude at which the burst ignites. As will be discussed in more detail below, burst ignition is most likely to take place on 
the stellar equator. Bhattacharyya \& Strohmayer (2006a,b) conjectured that rare high latitude ignition could lead to a doublepeaked burst if the burning front slows and partially stalls as it approaches the equator. This leads to a dip in the lightcurve: the second peak forms as the burning front recovers and accelerates again. The authors illustrated the concept by modelling two of the double-peaked bursts of $4 U$ 1636-536. A particularly attractive feature of this model is that it attempts to explain simultaneously both the spectral evolution and the detectability of millisecond oscillations during the burst rise.

So what determines ignition latitude? The general assumption is that accreting material comes into corotation and hydrostatic equilibrium on a timescale short compared to the recurrence time between bursts. The material must therefore spread to form an equipotential surface that on a rapidly rotating neutron star is oblate, since the rotation acts to reduce the effective gravity at the equator. This means that the local accretion rate varies with latitude, being higher at the equator. Spitkovsky et al. (2002) argued that this favoured equatorial ignition since it is here that the column depth necessary to trigger a burst is reached most quickly.

The issue of ignition latitude has now been revisited in more detail by Cooper \& Narayan (2007) (hereafter CN07). These authors start by assessing the implications of a local accretion rate above which burning stabilises (Bildsten 1998). As global accretion rate rises, there must come a point where the equator has stabilised but higher latitudes have not. In this case, ignition will occur off-equator. The situation is complicated by the existence of different regimes of unstable burning, but detailed modelling by $\mathrm{CN} 07$ confirms the general principle. Ignition occurs at the equator until a certain critical accretion rate (the precise value of which depends on the stellar parameters and the accreted composition). Above this rate, however, the ignition point moves rapidly towards the pole and remains there until the global accretion rate is so high that all bursting activity is suppressed.

The accretion rate dependence of the multi-peak bursts may therefore enable us to distinguish between the various mechanisms. If the multi-peaked bursts are indeed caused by polar ignition, for example, the results of $\mathrm{CN} 07$ indicate that we should expect to find them at higher global accretion rates than the single-peaked bursts. In Sect. 2 we present an analysis of the accretion rate dependence of the multi-peak bursts. The implications for the various models are discussed in Sect. 3.

\section{Observations and data analysis}

To test the accretion rate dependence of the multi-peak bursts seen with RXTE we can make use of the RXTE burst catalogue (Galloway et al. 2007), hereafter G07. This catalogue records, for over 1000 bursts, a complete set of burst parameters including two that can be used to assess accretion rate. The first is position in the colour-colour diagram. Sources like 4U 1636-536, known as atoll sources, trace out a path in the colour-colour diagram (Hasinger \& van der Klis 1989). One can assign a colourcolour coordinate $S_{z}$ that traces progress though the diagram and is thought to be proportional to global accretion rate (Fig. 4 of $\mathrm{G} 07)^{1}$. A precise calibration of this relationship, however, is difficult. The second measure that is used to estimate accretion rate is the persistent luminosity. This is considered to be less reliable than $S_{z}$, but its advantage is that it can be used to calibrate the

\footnotetext{
1 For atoll sources the colour-colour coordinate is usually called $S_{a}$ rather than $S_{z}$ (which is used for the higher accretion rate $\mathrm{Z}$ sources). In this paper, however, we use $S_{z}$ for consistency with G07.
}

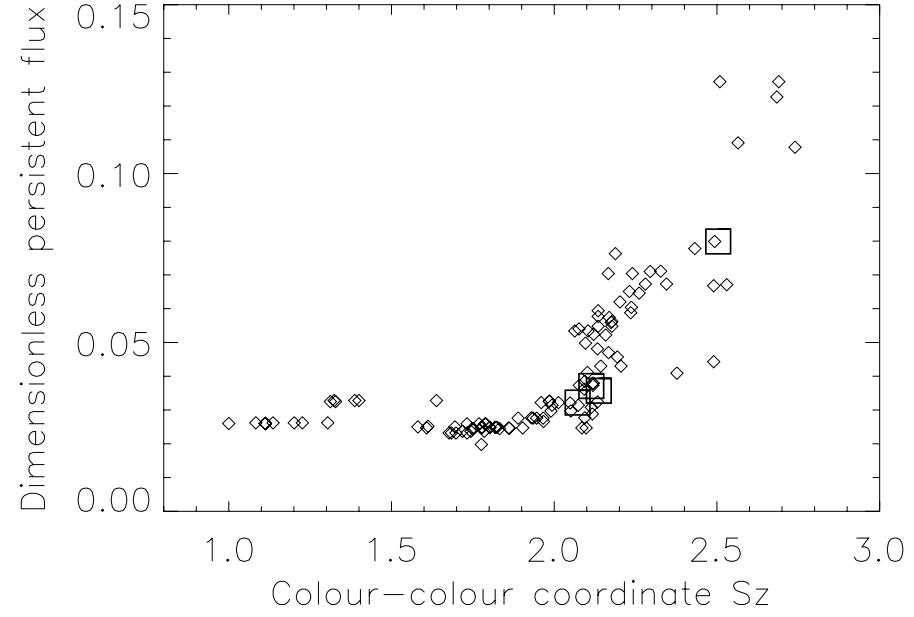

Fig. 2. Dimensionless persistent flux (as a fraction of the Eddington rate) against colour-colour diagram coordinate $S_{z}$ for X-ray bursts from $4 \mathrm{U}$ 1636-536, data from the RXTE burst catalogue (G07). $S_{z}$ is assumed to be proportional to accretion rate. Single peaked bursts are shown as diamonds, and the four multi-peaked non-PRE bursts by squares. Bursts (1)-(3) from Fig. 1 cluster together; Burst (4) sits alone.

accretion rate as a fraction of $\dot{M}_{\text {Edd }}$ provided that the distance is known (from, for example, a PRE burst). For a full discussion of these measures we refer the reader to Sect. 2.1 of G07.

We start by considering $4 U$ 1636-536, the source with the largest sample of multi-peak bursts. The RXTE catalogue contains 123 bursts from this source. Using PRE bursts as standard candles, G07 estimate that bursts are seen for accretion rates in the range $2-13 \% \dot{M}_{\text {Edd }}$. Figure 2 shows the distribution of singlepeaked and multi-peaked bursts in a plot of persistent flux (normalised by the Eddington flux) against $S_{z}$.

Figure 2 shows that the multi-peaked bursts do not appear at the highest inferred accretion rates. There are, even for the most extreme burst (burst number (4) in Fig. 1) single-peaked bursts at higher accretion rates. The EXOSAT data for $4 \mathrm{U} 1636-$ 536 support these findings. In May 1984 Sztajno et al. (1985) made a day-long observation during which the persistent flux declined by $\approx 30 \%$. Eight bursts were detected, four of which were double-peak non PRE bursts. Although the bursts were seen when the flux was relatively high, two single-peak bursts were also detected at this time. Later analysis by Prins \& van der Klis (1997) showed that at this time the source was in the middle banana state $\left(2.0<S_{z}<2.5\right)$, not the higher accretion rate upper banana state. The later double and triple-peaked bursts reported by van Paradijs et al. (1986) and Lewin et al. (1987) were also seen in a sequence of over 20 other bursts, at a time when the flux was rising. Although both were seen towards the higher end of the observed flux, there are other bursts in the sequence at higher fluxes that are single-peaked. Colour-colour diagram position is similar to the 1984 observations: by this measure too there are single-peaked bursts at higher accretion rates (van der Klis et al. 1990).

What about the other sources that have exhibited non-PRE double-peaked bursts? For $4 \mathrm{U}$ 1608-52 there is one multi-peak burst in the EXOSAT sample (Penninx et al. 1989) and one in the RXTE catalogue (Burst 24 from this source, G07 Fig. 9). Kuulkers et al. (2002) and Jonker et al. (2004) discovered one multi-peak burst apiece during RXTE observations of GX 17+2 and 4U 1709-267. Figure 9 of G07 also reveals previously unreported multi-peak non PRE bursts from Aql X-1 (Bursts 7 and 


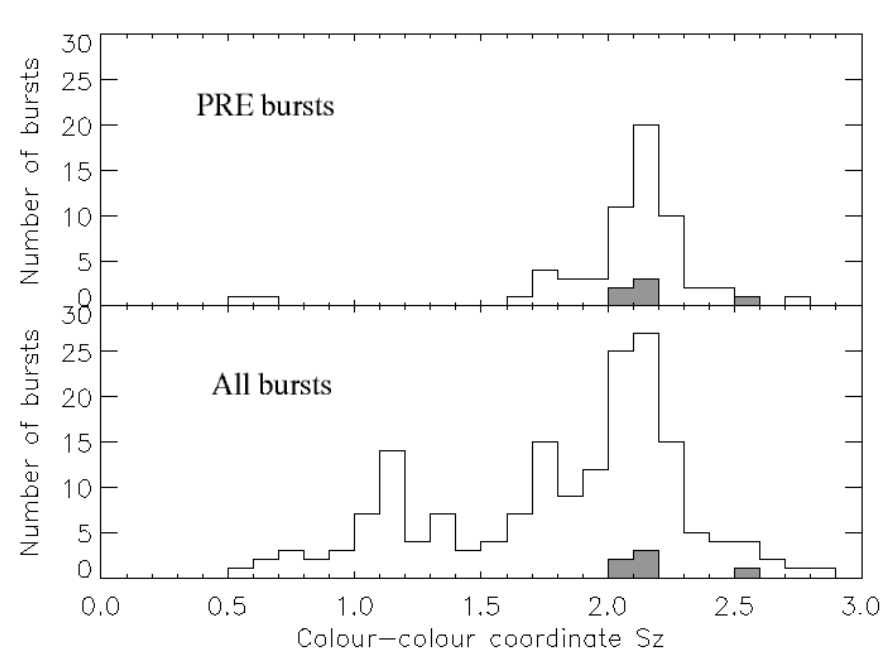

Fig. 3. The distribution of multi-peaked bursts (shaded) with $S_{z}$ compared to other bursts for the three sources in the RXTE catalogue that have a) non PRE multipeak bursts and b) trace out a long enough path in the colour-colour diagram to calculate $S_{z}$. These sources are 4U 1636536, Aql X-1 and 4U 1608-52. The lower panel shows the distribution compared to all other bursts, the upper panel the distribution compared to PRE bursts.

32) and EXO 0748-676 (Burst 82). Every single one of these sources has single-peaked bursts at higher accretion rates.

Given this sample, what can we say about the accretion rate at which multi-peak bursts occur? Three of the sources in the RXTE catalogue with multi-peak bursts trace out a full enough path in the colour-colour diagram to allow computation of $S_{z}$ : $4 \mathrm{U}$ 1636-536, 4U 1608-52 and Aql X-1 (although for the latter $S_{z}$ can only be calculated for one of the two multi-peak bursts). Figureé3 compares the distribution of the multi-peak and singlepeaked bursts with $S_{z}$ for these three sources. Five of the six bursts cluster around $S_{z}=2.1$, but the remaining burst (Burst 4 from Fig. 1) occurs at $S_{z} \approx 2.5$. The multi-peak non PRE bursts do seem to be associated with higher accretion rates: a two-sided Kolmogorov-Smirnov test gives only a $4 \%$ probability of them being drawn from the same underlying distribution as the set of all other bursts. The distribution of multi-peak bursts is however completely consistent with the distribution of PRE bursts, which are also associated with higher accretion rates. However, the sample of multi-peaked bursts is small, so we should treat these statistics with some caution.

We can also consider the dependence on absolute accretion rate as inferred from the persistent flux for those sources with a reasonable estimate of distance. 4U 1636-536 exhibits bursts at accretion rates from $2-13 \% \dot{M}_{\text {Edd }}$. Three of the multipeak bursts (Bursts (1)-(3) in Fig. 1) occur at accretion rates in the range 3.2 to $3.7 \% \dot{M}_{\text {Edd }}$, while the other (Burst (4) in Fig. 1) occurs at $8 \% \dot{M}_{\text {Edd. }}$ 4U 1608-52 has bursts for accretion rates in the range $0.5-10 \% \dot{M}_{\mathrm{Edd}}$, with the multipeak burst occurring at $3 \% \dot{M}_{\text {Edd }}$. Aql X-1 shows bursts for accretion rates in the range $0.1-11 \% \dot{M}_{\text {Edd }}$. The two multipeak bursts are at $2.3 \% \dot{M}_{\text {Edd }}$ and $3.3 \% \dot{M}_{\text {Edd }}$. EXO $0748-676$ has only been seen to burst at much lower accretion rates, in the range $0.5-1.5 \% \dot{M}_{\text {Edd }}$. The multipeak burst occurs at $0.9 \% \dot{M}_{\text {Edd }}$. For $4 U$ 1709-267 no calibration of accretion rate is possible as there are no definite PRE bursts. The multipeak burst from GX $17+2$ is particularly unusual since this source is a $\mathrm{Z}$ rather than an atoll source and accretes at a rate apparently in excess of $\dot{M}_{\text {Edd }}$. However, the morphology of this burst does differ from the other examples.

\section{Discussion}

The recent modelling by CN07 indicates that polar ignition should only take place above a certain accretion rate. If multipeak bursts are triggered by polar ignition, as suggested by Bhattacharyya \& Strohmayer (2006a,b), it follows that they should occur at higher accretion rates than the single-peaked bursts. This is clearly not the case. The bursts of $4 \mathrm{U} 1636-$ 536 also pose one other problem for the polar ignition model. Bhattacharyya \& Strohmayer (2006a,b) model bursts (3) and (4) in Fig. 1. For burst (3) they infer polar ignition, while for burst (4) they infer intermediate latitude ignition. Within the model of $\mathrm{CN} 07$, this would require burst (4) to appear at lower accretion rates than burst (3). The data show the exact opposite: burst (4) has a higher apparent accretion rate than bursts (1)-(3). So where does this leave the polar ignition model?

\subsection{Are multiple peaks a unique signature of polar ignition?}

The polar ignition model could still be viable if the majority of polar ignition bursts are single-peaked. In this scenario all of the bursts of $4 \mathrm{U} 1636-536$ for $S_{z} \gtrsim 2$ would be polar ignition bursts. If this were the case, there are several issues to be resolved. Firstly, this would set the accretion rate for the transition from equatorial to polar ignition at a few percent of $\dot{M}_{\text {Edd }}(\mathrm{G} 07$, and Fig. 2). The modelling of CN07 suggests however that this limit should be higher: in an example with a neutron star spinning at $650 \mathrm{~Hz}, \mathrm{CN} 07$ find a transition accretion rate of $\approx 16 \% \dot{M}_{\text {Edd }}$. The spin rate of $4 \mathrm{U} 1636-536$ is lower, at $580 \mathrm{~Hz}$ (Strohmayer et al. 1998), which would push the expected transition rate up. This disparity would need to be resolved. However, as we have discussed above, precise calibration of $S_{z}$ and accretion rate is difficult, and there are other areas of burst physics where similar discrepancies arise.

We must then consider the reason why a few, but not the vast majority, of the bursts are multi-peaked: the stalling mechanism. Bhattacharyya \& Strohmayer (2006a,b), revisiting an idea originally suggested by Sztajno et al. (1985), proposed that stalling is caused by the interaction of the burning front with a spreading layer of accreted material (Inogamov \& Sunyaev 1999). They suggest that stronger bursts may be able to disrupt this spreading layer and overcome the stalling mechanism, giving rise to single-peaked bursts. If this is the case, we would expect the fluence of the multi-peak bursts to be lower than that of other bursts in the same region of the colour-colour diagram. Figure 4 shows that although the multi-peak bursts do have low fluences, there are single-peaked bursts at similar accretion rates with fluences that are the same or even lower. For the polar ignition model to survive it would be necessary to explain these low fluence singlepeaked bursts. One possibility might be that these lower fluence bursts are so weak that the burning front stalls completely at the equator and never reaches the second hemisphere at all.

\subsection{Do other factors affect the latitude dependence of ignition?}

The other possibility is that the models of CN07 do not fully characterize the problem. As the authors themselves note, whilst their models do reproduce, for example, average burst recurrence times, they do not necessarily reproduce the scatter around this average. There are clearly subtleties waiting to be resolved (see also the discussion in Cooper \& Narayan (2006)). CN07 assume, for example, that variations in the conditions on the stellar surface are set only by the effective gravitational potential. We can 


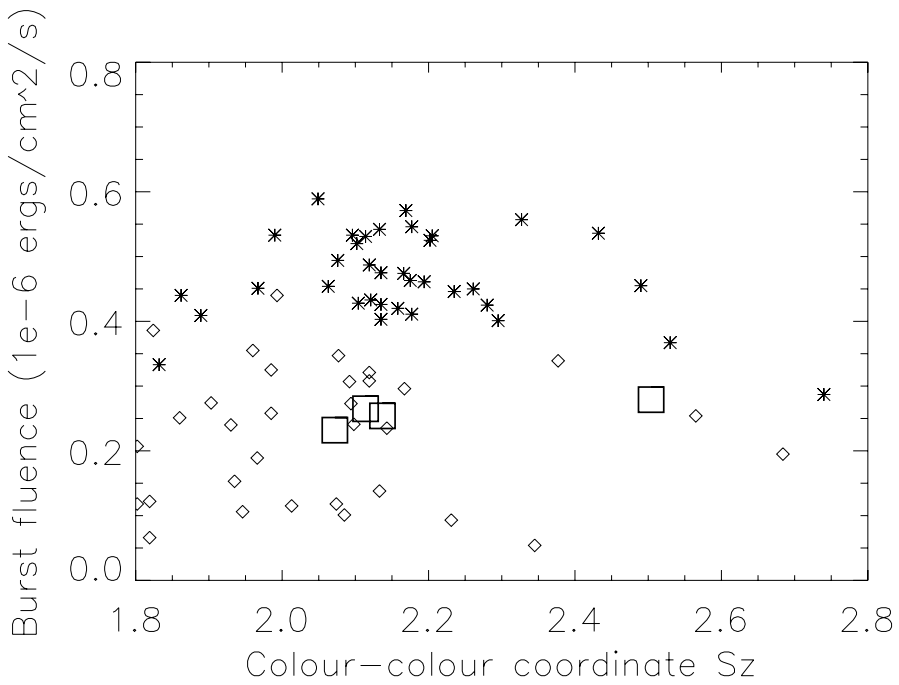

Fig. 4. Burst fluence against accretion rate as measured by $S_{z}$ (data taken from G07). Diamonds: single peaked non-PRE bursts; Asterisks: PRE bursts; Squares: multi-peak non PRE bursts. Error bars are similar in size to the diamonds.

break this assumption down into several elements. Firstly, that accreted fuel spreads rapidly and is not impeded in any way. Secondly that a similar re-adjustment occurs after a burst if any unburnt fuel remains. Thirdly, that there is no additional asymmetry that would affect burst triggering. If any of these conditions were violated, they might permit polar and equatorial ignition to co-exist at the same accretion rate.

The assumption of fuel spread, based on estimates of its viscosity, is hard to dispute (Bildsten 1998). Magnetic confinement could lead to asymmetries in fuel distribution if the field were strong enough. However, there is no evidence for persistent pulsations in 4U 1636-536, suggesting that the magnetic field is in this case too weak to channel or confine fuel. What about additional sources of latitude-dependent asymmetry? Two effects that should perhaps be considered are (a) the effect on ignition conditions (not just stalling conditions) of a spreading layer at the equator (Inogamov \& Sunyaev 1999), and (b) latitudedependent temperature asymmetries. The latter could arise if, for example, burning fronts launched from the equator repeatedly stalled when approaching the pole (due to reduced flame speed, Spitkovsky et al. 2002). Reduced heat injection into the crust at high latitudes could lead to a temperature gradient that might affect subsequent ignition conditions. Latitude dependent heating due to either core fluid oscillations or slightly asymmetric accretion could also play a role. We note that both of these mechanisms have been discussed as a means of generating a quadrupole moment that might limit the spin of rapidly rotating neutron stars via gravitational wave emission (Ushomirsky et al. 2000).

\subsection{Alternatives to the polar ignition model}

If the polar ignition model cannot be reconciled with the observations, an alternative is required. The nuclear waiting point model of Fisker et al. (2004) predicts multi-peak bursts at accretion rates of a few percent $\dot{M}_{\mathrm{Edd}}$, in line with the observations. The existence of multiple waiting points in the rp process also offers a natural explanation for the triple peaked burst, something that is hard to explain in the polar ignition model. The alternative is two stage burning triggered by hydrodynamical instabilities (Fujimoto et al. 1988). Bhattacharyya \& Strohmayer (2006a) criticised this model on the grounds that it was hard to see how an unburnt layer of fuel could be maintained for long enough for the mechanism to work. However, a recent study by Galloway et al. (2006) of the PRE bursts of $4 \mathrm{U}$ 1636-536 suggested the presence of an additional source of stratification in this star: this may promote the survival of unburnt fuel layers. Theoretical effort is required, however, to generate models of spectral evolution and timing behaviour for these alternative mechanisms in order to subject them to rigorous test.

\section{Conclusions}

We have presented an analysis of the accretion rate dependence of multi-peaked non PRE bursts, focusing in particular on whether they occur at higher accretion rates than singlepeaked bursts. The fact that they do not contradicts one of the predictions of the polar ignition model, given the recent study by $\mathrm{CN} 07$ that predicts a strong dependence of ignition latitude on accretion rate. While there are ways in which the polar ignition/stalling model could be reconciled with the data, alternative models should also be pursued. Ultimately a combination of models may prove to be the answer, particularly given that the multi-peak bursts are not a homogeneous set. To see this one only has to consider the differences in morphology and apparent accretion rate for the four bursts in Fig. 1.

Acknowledgements. We thank Lars Bildsten, Tod Strohmayer, Randall Cooper, Henk Spruit, Rashid Sunyaev, Duncan Galloway and Stuart Sim for comments.

\section{References}

Bhattacharyya, S., \& Strohmayer, T. E. 2006a, ApJ, 636, L121

Bhattacharyya, S., \& Strohmayer, T. E. 2006b, ApJ, 641, L53

Bildsten, L. 1998, in The Many Faces of Neutron Stars, ed. R. Buccheri, J. van Paradijs, \& M. A. Alpar (NATO ASI Ser. C. 515; Dordrecht; Kluwer), 419

Cooper, R. L., \& Narayan, R. 2006, ApJ, 652, 584

Cooper, R. L. \& Narayan, R. 2007, ApJ, 657, L29

Cornelisse, R. et al. 2003, A\&A, 405, 1033

Fisker, J. L., Thielemann, F. K., \& Wiescher, M. 2004, ApJ, 608, L61

Fujimoto, M. Y., Hanawa, T., \& Miyaji, S. 1981, ApJ, 247, 267

Fujimoto, M. Y., Sztajno, M., Lewin, W. H. G., \& van Paradijs, J. 1988, A\&A, 199, L9

Fushiki, I. \& Lamb, D. Q. 1987, ApJ, 323, L55

Galloway, D. K., Psaltis, D., Muno, M. P., \& Chakrabarty, D. 2006, ApJ, 639, 1033

Galloway, D. K., Muno, M. P., Hartman, J. M., et al. 2007, ApJS, submitted [arXiv: astro-ph/0608259]

Hasinger, G., \& van der Klis, M. 1989, A\&A, 225, 79

Inogamov, N. A., \& Sunyaev, R. A. 1999, Astron. Lett., 25, 269

Jonker, P. G., Galloway, D. K., McClintock, J. E., et al. 2004, MNRAS, 354, 666 Kuulkers, E., Homan, J., van der Klis, M., Lewin, W. H. G., \& Méndez, M. 2002, A\&A, 382, 947

Lewin, W. H. G., Penninx, W., van Paradijs, J., et al. 1987, ApJ, 319, 893

Narayan, R., \& Heyl, J. S. 2003, ApJ, 599, 419

Paczyński, B. 1983, ApJ, 267, 315

Penninx, W., Damen, E., van Paradijs, J., \& Lewin, W. H. G. 1989, A\&A, 208, 146

Prins, S., \& van der Klis, M. 1997, A\&A, 319, 498

Spitkovsky, A., Levin, Y., \& Ushomirsky, G. 2002, ApJ, 566, 1018

Strohmayer, T. E., Zhang, W., Swank, J. H., White, N. E., \& Lapidus, I. 1998, ApJ, 498, L135

Strohmayer, T. E., \& Bildsten, L. 2006, in Compact stellar X-ray sources, ed. Lewin W. H. G. \& van der Klis, M., Cambridge Astrophysics Series 39 (Cambridge: University Press), 113

Sztajno, M., van Paradijs, J., Lewin, W. H. G., et al. 1985, ApJ, 299, 487

Ushomirsky, G., Bildsten, L., \& Cutler, C. 2000, Proceedings of the 3rd Eduardo Amaldi Conference, AIP Conf. Proc. 523, 65

van der Klis, M., Hasinger, G., Damen, E., et al. 1990, ApJ, 360, L19

van Paradijs, J., Sztajno, M., Lewin, W. H. G., et al. 1986, MNRAS, 221, 617 